\title{
BROWNIAN MOTION AND A CONSEQUENCE OF HARNACK'S INEQUALITY: NODES OF QUANTUM WAVE FUNCTIONS ${ }^{1}$
}

\author{
M. HOFFMANN-OSTENHOF, T. HOFFMANN-OSTENHOF AND B. SIMON
}

\section{Abstract. Using Brownian motion ideas, we give two proofs that for a large class} of $V$ 's, $(-\Delta+V) u=E u$ and $u\left(x_{0}\right)=0$ implies that $u$ changes sign near $x_{0}$.

1. Introduction. In this paper we will consider potentials on $R^{\nu}$ of the form

$$
V(x)=\sum_{i=1}^{n} V_{i}\left(\pi_{i} x\right)
$$

with

$$
\pi_{i} \text { a linear map of } R^{\nu} \text { to } R^{\mu_{i}}
$$

and

$$
V_{i} \in L_{u}^{p}\left(R^{\mu_{i}}\right), \quad p>\mu_{i} / 2, p \geqslant 1 \text {. }
$$

Here $L_{u}^{p}$ is the set of uniformly local $L^{p}$ functions, i.e. functions with

$$
\sup _{x}\left[\int_{|y-x|<1}|V(y)|^{p} d y\right]<\infty .
$$

The complication of the $\pi_{i}$ 's is included to allow multiparticle potentials, $V\left(r_{1}, \ldots, r_{n}\right)=\sum W_{i j}\left(r_{i}-r_{j}\right) ; r_{i} \in R^{3}$ with $W_{i j}$ only required to be uniformly locally $L^{p}$ with $p>3 / 2$ rather than $p>3 n / 2$. The uniformly local functions are used to accomodate periodic potentials.

Our main goal in this paper will be the following result.

THEOREM 1.1. Let $V$ obey (1.1)-(1.3). Let $u \in L^{\infty}\left(R^{\nu}\right)$ be real and obey

$$
(-\Delta+V) u=0 \text {. }
$$

Suppose that $u\left(x_{0}\right)=0$. Then either

(a) $u$ is identically zero near $x_{0}$ or

(b) $u$ has both signs arbitrarily close to $x_{0}$.

REMARKS. (1) (1.4) is required in the sense of distributions. Equivalently, under the hypothesis, $H \equiv-\Delta+V$ as a form sum defines a semibounded operator on $L^{2}$

Received by the editors December 10, 1979.

AMS (MOS) subject classifications (1970). Primary 35J10, 60J65; Secondary 35B05, 81 A81.

Key words and phrases. Nodes, Brownian motion.

${ }^{1}$ Work supported in part by Fonds zur Förderung der wissenschaftlichen Forschung in Österreich, Project No. 3655 and U. S. NSF Grant MCS-78-01855. 
and $e^{-t H}$ extends by density as an exponentially bounded semigroup on $L^{p}$ $(p<\infty)$ and then by duality on $L^{\infty}[8]$. (1.4) thus holds in the sense that $e^{-t H} u=u$ for all $t$.

(2) It is known [8] under the stated hypothesis that any $L^{2}$ eigenfunction lies in $L^{\infty}$ and any $L^{\infty}$ eigenfunction is continuous. In particular, the condition $u\left(x_{0}\right)=0$ is meaningful and, moreover, the theorem will apply to both bound state eigenfunctions and suitable continuum eigenfunctions.

(3) The theorem asserts the nodal "surface" $\{x \mid u(x)=0\}$ must have (Hausdorff) dimension at least $\nu-1$.

(4) In many cases, it is known that (a) cannot occur if $u$ is not identically zero; for the locally bounded case, see [5]; for certain unbounded $V$ 's see [6]. The atomic case is included. Thus, in those cases, we can assert that (b) occurs.

For the case where $0=\inf \operatorname{spec}(H)$ and $u \in L^{2}$, it is known that $u$ is a.e. positive (Perron-Frobenius type result; see [5]) so that this theorem asserts that $u$ is everywhere nonvanishing. This special case is not new in the mathematical physics literature. For sufficiently nice $V$ 's, it was originally proven by Simon [7]. Carmona [1], [2] had the idea of using Brownian motion to prove such results; his results were mildly improved upon in [8] and, in particular, the special case for $V$ obeying (1.1)-(1.3) is to be found there.

Our methods here are motivated by Carmona's ideas. In $\$ 2$ we give a proof which is based on the idea that if neither (a) nor (b) is true, then $u$ will be nonnegative near $x_{0}$ and strictly positive in an open subset of this "near region". In a Feynman-Kac formula for $u\left(x_{0}\right)$, small times will involve mainly paths in the near region so $u\left(x_{0}\right)$ will be positive. In $\S 3$, we give an even slicker proof using stopping times.

There is another way of proving Theorem 1.1 using results in the P.D.E. literature. Trudinger [9] (see also Gilbarg and Trudinger [4]) has proven that for $V$ 's obeying (1.1)-(1.3), any $u$ obeying (1.4) with $u>0$ on some domain $\Omega \subseteq R^{\nu}$ satisfies a so-called weak Harnack inequality

$$
\sup _{x \in \Omega^{\prime}} u<C \inf _{x \in \Omega^{\prime}} u
$$

for every $\Omega^{\prime}$ strictly contained in $\Omega$. The constant $C$ depends on $\Omega^{\prime}, \Omega$ and $L^{p}(\Omega)$ properties of $V$. We caution the reader that in [4] and [9], conditions (1.1)-(1.3) are not mentioned, but, in a remark, Trudinger states that (1.5) holds under a suitable form-boundedness requirement on $V$ which is fulfilled for $V$ satisfying (1.1)-(1.3). We should also mention that (1.5) is a local result. In fact, the conditions on $V$ and $u$ need to be only stated locally (replace $L^{p}\left(R^{p}\right)$ by $L^{p}(\Omega)$ etc.).

Theorem 1.1 follows trivially from (1.5). It would be interesting to find a proof of (1.5) using Brownian motion ideas.

As for extensions of Theorem 1.1, we note that probabilistic methods and also Harnack inequality methods can be extended to a larger class of second order elliptic operators. Since $\Delta^{2}\left(x^{2}+y^{2}+z^{2}\right)=0$, the result will not hold for operators of order higher than two.

The third author would like to thank W. Thirring for the hospitality of the 
Institute for Theoretical Physics of the University of Vienna where this work was done.

2. First proof: small time estimates. Our first proof exploits the intuition mentioned already in $\$ 1$. While we will freely use the language of Brownian motion (see e.g. [8]), one can give the proof without even mentioning Brownian motion using analytical ideas: the only really subtle point is a somewhat tricky use of the Hadamard three line lemma to replace Hölder's inequality in the path integral. For an example of the kind of translation we have in mind compare the discussion of $e^{-t H}: L^{2} \rightarrow L^{\infty}$ in [8] and that in [3].

We will need the following lemma from [8].

LEMMA 2.1. Let V obey (1.1)-(1.3). Then

$$
\sup _{x \in R^{n} ; 0<t<1} E\left(\exp \left\{-\int_{0}^{t} V(x+b(s)) d s\right\}\right)<\infty .
$$

In (2.1), $b$ is Brownian motion and $E$ its expectation value.

Without loss of generality we suppose $x_{0}=0$.

In both this proof and the next it is useful to note that if both (a) and (b) fail we can find positive $\varepsilon_{0}, r, \delta$ and a vector $x_{1}$ with

$$
\left|x_{1}\right|<r / 6, \quad \delta<r / 6
$$

so that

$$
u \geqslant 0 \text { on }\{x|| x \mid<r\}
$$

and

$$
u>\varepsilon_{0} \text { on }\left\{x|| x-x_{1} \mid<\delta\right\}
$$

To accomplish this we may need to replace $u$ by $-u$ and we use the continuity of $u$.

First Proof of Theorem 1.1. Let $\chi_{1}$ be the characteristic function of the set in (2.3), $\chi_{2}=1-\chi_{1}$ and $\chi_{3}$ the characteristic function of the set in (2.4). By the Feynman-Kac formula and $e^{t H} u=u$, we have that, for any $t$,

$$
\begin{aligned}
u(0) & =\left(e^{-t H} u\right)(0)=A_{t}+B_{t}, \\
A_{t} & =E\left(\exp \left\{-\int_{0}^{t} V(b(s)) d s\right\} u(b(t)) \chi_{1}(b(t))\right), \\
B_{t} & =E\left(\exp \left\{-\int_{0}^{t} V(b(s)) d s\right\} u(b(t)) \chi_{2}(b(t))\right) .
\end{aligned}
$$

By hypothesis, $A_{t}>0$ and we will show that for $t$ small $\left|B_{t}\right|<A_{t}$. This contradicts the assumption that $u(0)=0$ and completes the proof. By the Schwarz inequality

$$
\begin{aligned}
\left|B_{t}\right| & \leqslant\|u\|_{\infty} E\left(\chi_{2}(b(t))\right)^{1 / 2} E\left(\exp \left\{-2 \int_{0}^{t} V(b(s)) d s\right\}\right)^{1 / 2} \\
& \leqslant \tilde{C} E\left(\chi_{2}(b(t))\right)^{1 / 2} \leqslant C_{1} \exp \left\{-r^{2} /(1+\varepsilon) 4 t\right\}
\end{aligned}
$$

for all $t \in(0,1]$. Here $C_{1}$ is $\varepsilon$ (and $r$ ) dependent. The first step uses the lemma and the second, the explicit Gaussian distribution of $b(t)$. 
On the other hand, we note that $E\left(F^{2}\right) \geqslant|E(F G)|^{2} / E\left(G^{2}\right)$ with

$$
F=\exp \left\{-\int_{0}^{t} V(b(s)) d s\right\} \chi_{3}, \quad G=\exp \left\{\int_{0}^{t} V(b(s)) d s\right\}
$$

so that

$$
\begin{aligned}
A_{t} & \geqslant \varepsilon_{0} E\left(\exp \left\{-\int_{0}^{t} V(b(s)) d s\right\} \chi_{3}\right) \geqslant \varepsilon_{0} E\left(\chi_{3}\right)^{2} E\left(\exp \left\{\int_{0}^{t} V(b(s)) d s\right\}\right)^{-1} \\
& \geqslant C_{2} \exp \left\{-r^{2} / 9(1-\varepsilon) t\right\}
\end{aligned}
$$

where we used the lemma to get a lower bound on $\left[E\left(\exp \left\{\int_{0}^{t} V(b(s)) d s\right\}\right)\right]^{-1}$ and supp $\chi_{3} \subset\{x|| x \mid<r / 3\}$ and the explicit Gaussian in the last step. Thus, for $t$ small $A_{t}>\left|B_{t}\right|$.

\section{Second proof: Stopping times. Recall}

Definition. Let $f(t, b)$ be a function $[0, \infty) \times \mathscr{B}$, with $\mathscr{B}$ the set of Brownian paths. $f$ is called a supermartingale if and only if

(i) $f(t, b)$ is only a function of $\{b(s) \mid s \leqslant t\}$.

(ii) If $E(\cdot \mid\{b(s) \mid s \leqslant t\})$ is a conditional expectation, then for $w<t$

$$
E(f(t, \cdot) \mid\{b(s) \mid s<w\}) \leqslant f(w, \cdot) .
$$

Definition. A stopping time $\tau$ is a map from $\mathscr{B}$ to $[0, \infty)$ so that $\{b \mid \tau(b)<t\}$ is only a function of $\{b(s) \mid s \leqslant t\}$.

The following lemma is proven in [8] (it is fairly standard).

LEMMA 3.1. Let $f$ be a supermartingale with $\sup _{t, b}|f(t, b)|<\infty$. Then

$$
E(f(0, \cdot)) \geqslant E(f(\tau(b), b)) .
$$

REMARKs. (1) $f(0, b)$ is independent of $b$ and thus a number.

(2) The result is false if the sup-condition is dropped, see [8].

(3) The proof is very simple with the proper discrete approximation of $\tau$. The sup-condition comes in via a dominated convergence theorem which is used to remove the discretization.

Second Proof of Theorem 1.1. Let $V_{ \pm}=\max ( \pm V, 0)$. Define

$$
f(t, b)=\exp \left\{-\int_{0}^{t} V_{+}(x+b(s)) d s\right\} u(b(t))
$$

and for $w<t$

$$
g(w, t, b)=\exp \left\{-\int_{0}^{t} V_{+}(x+b(s)) d s\right\} \exp \left\{\int_{0}^{w} V_{-}(x+b(s)) d s\right\} u(b(t)) .
$$

Then $f(t, \cdot)$ is only a function of $\{b(x) \mid s<t\}$ and

$$
E(f(t, \cdot) \mid\{b(s) \mid x \leqslant w\})<E(g(w, t, b) \mid\{b(s) \mid s<w\})=f(w, \cdot)
$$

by the Markov property and the Feynman-Kac formula together with $e^{-(t-w) H} u=$ u. Clearly

so for any stopping time

$$
\sup _{t, b}|f(t, b)|<\|u\|_{\infty}<\infty
$$

$$
E(f(\tau(b), b))<E(f(0, \cdot))=u(0) .
$$


If the theorem fails, we may suppose that (2.2)-(2.4) hold. Let $\tau(b)=$ first time that $|b(t)|=\left|x_{1}\right|$. Then, by (2.3), $f(\tau(b), b)>0$ for all $b$. Moreover, by the lemma below and the fact that $\left|b(\tau(b))-x_{1}\right|<\delta$ on a set of nonzero measure, $f(\tau(b), b)$ $>0$ on a set of nonzero measure. Thus $E(f(\tau(b), b))>0$ violating (3.2).

LEMMA 3.2. For a.e. b,

$$
\int_{0}^{\tau(b)} V_{+}(b(s)) d s<\infty .
$$

Proof. Since $\tau(b)<\infty$, a.e. it suffices that $\int_{0}^{t} V(b(s)) d s<\infty$ a.e. $b$ for each fixed $t$. But this is proven in [8].

Note ADDED IN PROOF. A Brownian motion proof of (1.5) has been found (M. Aizenman and B. Simon, in preparation).

\section{REFERENCES}

1. R. Carmona, Pointwise bounds for Schrödinger eigenstates, Comm. Math. Phys. 62 (1978), 97-106.

2. 259-296.

3. P. Deift, W. Hunziker, B. Simon and E. Vock, Pointwise bounds on eigenfunctions and wavepackets in n-body quantum systems. IV, Comm. Math. Phys. 64 (1978), 1-34.

4. D. Gilbarg and N. S. Trudinger, Elliptic partial differential equations of second order, Grundlehren der Mathematischen Wissenschaften, Vol. 224, Springer-Verlag, Berlin-New York, 1977.

5. M. Reed and B. Simon, Methods of modern mathematical physics. IV. Analysis of operators, Academic Press, New York, 1978.

6. M. Schechter and B. Simon, Unique continuation for Schrödinger operators with unbounded potentials, J. Math. Anal. Appl. (to appear).

7. B. Simon, Pointwise bounds on eigenfunctions and wave packets in n-body quantum systems. III, Trans. Amer. Math. Soc. 208 (1975), 317-329.

8. __ Functional integration and quantum physics, Academic Press, New York, 1979.

9. N. S. Trudinger, Linear elliptic operators with measurable coefficients, Ann. Scuola Norm. Sup. Pisa Sci. Fis. Mat. (3) 27 (1973), 265-308.

Institut für Theoretische Physik, Universität Wien, A-1090 Wien, Austrin

Institut für Theoretische Chemie und Strahlenchemie, Universität Wien, A-1090 Wien, Austrin

Departments of Mathematics and Physics, Princeton University, Prnnceton, New Jersey 08544 